\title{
Brownian dynamics study of dynamic scaling and related freezing criteria in quasi-two-dimensional dispersions
}

\author{
Raphaël Pesché and Markus Kollmann \\ Fachbereich Physik, Universität Konstanz, Postfach 5560, D-78457 Konstanz, Germany \\ Gerhard Nägele ${ }^{\text {a) }}$ \\ Institut für Festkörperforschung, Forschungszentrum Jülich, D-52425 Jülich, Germany
}

(Received 30 January 2001; accepted 27 February 2001)

\begin{abstract}
We present a Brownian dynamics simulation study of static and dynamic properties of quasi-two-dimensional dispersions of colloidal spheres interacting by long-range screened electrostatic and by dipolar magnetic forces, respectively. The calculated van Hove real-space dynamic correlation functions, mean squared displacements, and hydrodynamic functions are shown to obey a dynamic scaling behavior in terms of a characteristic relaxation time related to the geometrical mean particle distance. Hydrodynamic interactions introduce a second characteristic length scale, and they lead to a more restricted scaling behavior with an enhancement of self-diffusion. As a consequence of dynamic scaling, the dynamical criterion of Löwen [Phys. Rev. E 53, R29 (1996)] for the onset of colloidal freezing is shown to be equivalent to a two-dimensional freezing criterion related to the static structure factor. (C) 2001 American Institute of Physics.
\end{abstract}

[DOI: $10.1063 / 1.1366637]$

\section{INTRODUCTION}

During the last several years, a lot of experimental and theoretical effort has been devoted to studying the static and dynamic properties of quasi-two-dimensional (Q2D) dispersions of colloidal particles. Experimentally well-studied examples of such dispersions are charged colloidal particles confined between two narrow glass plates,${ }^{1-3}$ and superparamagnetic colloidal particles suspended in water at a waterair interface (hanging-drop geometry) and interacting via repulsive dipolar magnetic forces due to an applied magnetic field. ${ }^{4}$ Part of the interest in these systems arises from the fact that, contrary to low-molecular liquids, the range, strength, and even the sign of the particle interactions can be experimentally controlled. Moreover, the particle trajectories of micron-sized colloidal particles can be directly tracked using video microscopy imaging. ${ }^{2}$

One area of intensive research is concerned with the nature of two-dimensional freezing and melting. Contrary to three-dimensional freezing, which is a first-order phase transition, two-dimensional freezing may be a two-stage process of continuous phase transitions with an intermediate hexatic phase of long-range orientational order. ${ }^{5}$ Strong experimental evidence for such a two-stage transition scenario has indeed been found very recently for magnetic particle monolayers with long-range dipolar repulsion. ${ }^{4}$

There is also considerable interest in understanding the effective pair potential between charged colloidal particles confined between two charged glass plates. Recent experiments show evidence of attraction between like-charged colloidal ions at intermediate distances. ${ }^{3}$ While some wallinduced electrostatic mechanisms have been discussed which may lead to attraction, ${ }^{6,7}$ it has appeared very recently that

\footnotetext{
a) Author to whom correspondence should be addressed; electronic mail: g.naegele@fz-juelich.de
}

some of the direct imaging measurements of particle attraction $^{3}$ can be attributed to a nonequilibrium hydrodynamic effect. ${ }^{8}$ In this context, it should be noted that, contrary to low-molecular liquids, the dynamics of colloidal dispersions is controlled not only by direct interparticle forces, but also by solvent-mediated hydrodynamic interactions (HI). Hydrodynamic interactions can give rise to remarkable dynamical effects ${ }^{9}$ like the enhancement of selfdiffusion $^{10-12}$ and the divergence of the collective diffusion coefficient related to the hydrodynamic function of Q2D systems at small wave numbers. ${ }^{13}$

This paper is concerned with the general dynamic behavior of quasi-two-dimensional dispersions of spherical particles with strong and long-range repulsive interactions. We employ a Brownian dynamics (BD) simulation method to analyze the static and dynamic scaling behavior of these quasi-two-dimensional systems, and to unravel its implications on related static and dynamic freezing criteria. Particular focus is given to reveal the influence of $\mathrm{HI}$ on the dynamic scaling. For this purpose, various measurable quantities are calculated in dependence on radial distance, wave number, correlation time, and interaction strength: radial distribution functions and static structure factors, and dynamic properties like mean squared displacements, longtime self-diffusion coefficients, van Hove real space correlation functions, and hydrodynamic functions. The importance of $\mathrm{HI}$ is assessed by comparing BD results where (far-field) $\mathrm{HI}$ are included with BD data where $\mathrm{HI}$ have been neglected.

\section{Q2D MODEL SYSTEMS AND BROWNIAN DYNAMICS METHOD}

Our BD study is focused on Q2D suspensions of spherical colloidal particles with strong and long-range repulsions where the excluded volume interactions are completely masked. Two types of effective pair potentials, $u(r)$, are 
considered: first a two-dimensional Yukawa-type potential of the form ${ }^{14}$

$$
\beta u(r)=L_{B} Z^{2} \frac{e^{-\kappa r}}{r}, \quad r>\sigma
$$

plus a hard core repulsive part for $r<\sigma$. Here, $r$ is the center to center distance between two charged colloidal spheres of diameter $\sigma=2 a, Z$ is an effective particle charge in units of the elementary charge, $\beta=1 / k_{B} T$, and $L_{B}$ is the so-called Bjerrum length of the solvent $(=7.2 \AA$ for water at room temperature). Equation (1) has been used in various simulation studies as a model potential for the lateral interaction of charged colloidal spheres located between two closely spaced planar glass plates, with the screening length, $\kappa^{-1}$, determined essentially by the counterions dissociated from the highly charged glass plates. ${ }^{13,15,16}$ The potential in Eq. (1) contains $\kappa^{-1}$ as an intrinsic length scale.

The second potential used in our BD study is a repulsive dipolar potential of the form

$$
\beta u(r)=\frac{\mu_{0}}{4 \pi k_{B} T} \frac{M^{2}}{r^{3}} .
$$

This potential describes, to very good accuracy, the magnetic interactions of superparamagnetic colloidal spheres of induced magnetic moment $M=\chi_{\text {eff }} B$, located at a liquid-air interface, where a magnetic field of strength $B$ is applied perpendicular to the interface. Here, $\chi_{\text {eff }}$ is the effective magnetic susceptibility of the particles. Such well-defined quasitwo-dimensional systems have been studied experimentally and theoretically in Refs. 17 and 12. Contrary to Eq. (1), the magnetic pair potential in Eq. (2) has no intrinsic length scale.

We compute the in-plane overdamped diffusion of the colloidal spheres interacting by the pair potentials (1) and (2), respectively, by using a BD simulation method. This method allows one to generate numerically the twodimensional trajectories $R_{i}(t)$ of the colloidal particles. It is statistically equivalent to solving the generalized Smoluchowski equation for the many-particle probability density function.

The BD finite difference equation describing the inplane displacement of $N$ identical spherical Brownian particles immersed in a fluid of viscosity $\eta$ during the time step $\Delta t$ is given by ${ }^{12,13,18}$

$$
\begin{aligned}
\mathbf{R}_{i}(t+\Delta t)-\mathbf{R}_{i}(t)= & \sum_{j=1}^{N}\left[\beta \mathbf{D}_{i j}\left(\mathbf{R}^{N}\right) \cdot \mathbf{F}_{j}^{P}+\nabla_{j} \cdot \mathbf{D}_{i j}\left(\mathbf{R}^{N}\right)\right] \Delta t \\
& +\Delta \mathbf{X}_{i}+\mathcal{O}\left(\Delta t^{2}\right) .
\end{aligned}
$$

Here, $\mathbf{R}_{i}(t)$ is the position vector pointing to the center of sphere $i$ at time $t, \mathbf{F}_{j}^{P}$ is the direct total force on particle $j$ due to all other $N-1$ particles, as derived from the pair potentials of Eqs. (1) and (2), respectively, and $\Delta \mathbf{X}_{i}$ is a random displacement vector of particle $i$ originating from solvent particle collisions. The random displacement $\Delta \mathbf{X}_{i}$ is sampled from a Gaussian distribution with zero mean, i.e.,

$$
\left\langle\Delta \mathbf{X}_{i}\right\rangle=0
$$

and covariance matrix

$$
\left\langle\Delta \mathbf{X}_{i} \Delta \mathbf{X}_{j}\right\rangle=2 \mathbf{D}_{i j}\left(\mathbf{R}^{N}\right) \Delta t .
$$

The angular brackets indicate an equilibrium ensemble average and we employ here the dyadic tensor notation.

The solvent-mediated HI between particles $i$ and $j$ are accounted for in the BD algorithm through the hydrodynamic diffusivity tensors $\mathbf{D}_{i j}\left(\mathbf{R}^{N}\right)$ which depend, in principle, on the configuration $\mathbf{R}^{N}=\left\{\mathbf{R}_{1}, \ldots, \mathbf{R}_{N}\right\}$ of all $N$ sphere centers at time $t$. For the strongly repelling and rather dilute dispersed colloidal particles under consideration, HI are dominated by their pairwise additive long-range part. Therefore, we can treat $\mathbf{D}_{i j}$ within good accuracy on the Rotne-Prager (RP) approximation level, i.e.,

$$
\mathbf{D}_{i j}\left(\mathbf{R}^{N}\right) \approx D_{0}\left[\delta_{i j} \mathbf{1}+\left(1-\delta_{i j}\right) \mathbf{T}^{\mathrm{RP}}\left(\mathbf{R}_{i}-\mathbf{R}_{j}\right)\right],
$$

where $\mathbf{1}$ is the unit tensor, $\delta_{i j}$ is the Kronecker symbol, and $D_{0}$ denotes the in-plane free diffusion coefficient of a particle. The Rotne-Prager tensor, $\mathbf{T}^{\mathrm{RP}}$, reads explicitly ${ }^{19}$

$$
\mathbf{T}^{\mathrm{RP}}(\mathbf{r})=\frac{3}{4}\left(\frac{a}{r}\right)[\mathbf{1}+\hat{\mathbf{r}} \hat{\mathbf{r}}]+\frac{1}{2}\left(\frac{a}{r}\right)^{3}[\mathbf{1}-3 \hat{\mathbf{r}} \hat{\mathbf{r}}],
$$

with $\hat{\mathbf{r}}=\mathbf{r} / r$. It consists of a monopole (Oseen) contribution proportional to $r^{-1}$, and of a dipolar part proportional to $r^{-3}$. On the RP approximation level, the positive definiteness of the exact diffusivity tensor matrix is preserved. ${ }^{19,20}$

In principle, the value of the in-plane free diffusion coefficient $D_{0}$ depends on hydrodynamic flow geometry parameters like the distance of the spherical colloid from the air-water free interface in the case of superparamagnetic particles ${ }^{21}$ and on the separation of the parallel glass plates in the case of charged particles located in between. ${ }^{8,13}$ For instance, the free diffusion coefficient, $D_{0, f}$, of an isolated sphere translating without rotation parallel to, and almost in contact with, the water-air interface is given approximately by $1.35 D_{0}$, where $D_{0}$ is the Stokesian free diffusion coefficient in the bulk liquid. ${ }^{22}$ Stokesian dynamics simulation results with near-field $\mathrm{HI}$ included as well as experimental data for the free diffusion coefficient, $D_{0, h}$, of a sphere translating in the midplane between two parallel walls separated by a distance $h$ apart from each other have been reported in Refs. 13 and 23, respectively. However, since we consider here only diffusional quantities normalized, respectively, by $D_{0 . f}$ and $D_{0, h} \leqslant D_{0}$, the actual values of these zero-density coefficients is of no concern. Moreover, the forms of the multipole moments in the far-field expression of the two-body diffusivity tensor $\mathbf{D}_{i j}$ may depend on the Q2D system geometry and on the boundary conditions considered. The leading asymptotic far-field form of $\mathbf{D}_{i j}$ for two particles located right at the liquid-gas interface is given by the monopole (i.e., Oseen) contribution in Eqs. (6) and (7), but with $D_{0}$ replaced by $D_{0, f} \cdot{ }^{24-26}$ This far-field term dominates the HI of the superparamagnetic particles under consideration (cf., e.g., Fig. 6). The leading order far-field form of $\mathbf{D}_{i j}$ for the lateral motion of two particles between two parallel walls depends, of course, on the plate distance $h \cdot{ }^{13,27-29}$ However, we are not concerned here with the variation of the lateral diffusion coefficient with the plate separation (i.e., we keep $h$ fixed), but with the general static and dynamic scaling rela- 
tions in two dimensions and their implications. The general qualitative influence of $\mathrm{HI}$ on the scaling relations discussed in the following can be expected to be independent of the chosen wall distance, at least as long as strong wall lubrication effects are absent. For these reasons, we use for simplicity the far-field RP form of $\mathbf{D}_{i j}$ as given in Eqs. (6) and (7), which corresponds to an unbounded solvent.

In our simulations, typically $N=100-2500$ particles confined in a periodically repeated square were equilibrated using a $N V T$-ensemble Monte Carlo method. After equilibration, several thousand production time steps were generated by the $\mathrm{BD}$ algorithm in Eq. (3) to obtain various diffusional and structural properties (cf. the following). The calculations have been speeded up using Verlet next neighbors lists and interpolation of stored force values. The longrange nature of $\mathrm{HI}$ is accounted for by employing an Ewaldtype summation technique on the Rotne-Prager level as developed by Beenakker. ${ }^{30}$ The influence of HI on dynamic properties is analyzed through comparison with $\mathrm{BD}$ calculations where $\mathrm{HI}$ are disregarded, i.e., where $\mathbf{D}_{i j}=D_{0} \mathbf{1} \delta_{i j}$. In Eq. (3), we use a time step of $\Delta t=10^{-5}-10^{-4} \tau_{0}$ where $\tau_{0}$ $=1 /\left(n D_{0}\right)$ is the time needed for a free particle to diffuse the geometrical mean interparticle distance $r_{0}=n^{-1 / 2}$, and $n$ is the two-dimensional (areal) number density. The accuracy of our BD simulation method was tested for specific examples by comparison with published simulation data on Q2D systems. ${ }^{12}$

The following static and dynamic properties of charged and magnetic Q2D systems characterized by the pair potentials in Eqs. (1) and (2), respectively, are calculated: lateral static pair correlations between two particles a distance $r$ apart from each other as described by the radial distribution function

$$
g(r)=\frac{1}{n}\left\langle\frac{1}{N} \sum_{\substack{i, j=1 \\ i \neq j}}^{N} \delta\left(\mathbf{r}-\mathbf{R}_{i}+\mathbf{R}_{j}\right)\right\rangle .
$$

The function $g(r)$ is related to the corresponding twodimensional static structure factor $S(q)$ by the FourierBessel transform

$$
S(q)=1+2 \pi n \int_{0}^{\infty} d r r[g(r)-1] J_{0}(q r),
$$

where $q$ is the scattering wave number and $J_{0}$ is the zerothorder Bessel function of the first kind.

A key quantity describing the self-diffusion of a particle $i$ is the lateral mean-squared displacement $W(t)$, defined by

$$
W(t)=\frac{1}{4}\left\langle\frac{1}{N} \sum_{i=1}^{N}\left[\mathbf{R}_{i}(t)-\mathbf{R}_{i}(0)\right]^{2}\right\rangle .
$$

The function $W(t)$ exhibits a linear time dependence at short and long times, i.e., $W(t) \approx D_{S}^{S} t$ for $t \ll \tau_{0}$ and $W(t) \approx D_{S}^{L} t$ for $t \gg \tau_{0}$, which defines the short-time and long-time selfdiffusion coefficients $D_{S}^{S}$ and $D_{S}^{L}$, respectively.

Space-time correlations for a single sphere and for two colloidal spheres are described, respectively, by the dimen- sionless self-intermediate and distinct van Hove correlation functions $g_{s}(r, t)$ and $g_{d}(r, t) .{ }^{31}$ The self-part is given for an isotropic system by

$$
\begin{aligned}
g_{s}(r, t) & =\frac{1}{n}\left\langle\frac{1}{N} \sum_{i=1}^{N} \delta\left(\mathbf{r}-\mathbf{R}_{i}(t)+\mathbf{R}_{i}(0)\right)\right\rangle \\
& \approx \frac{1}{4 \pi n W(t)} e^{-r^{2} /[4 W(t)]} .
\end{aligned}
$$

The second approximate equality in Eq. (11) holds only provided that non-Gaussian contributions to $g_{s}(r, t)$ are very small. ${ }^{32,33}$ The function $g_{s}(r, t)$ is proportional to the conditional probability density that a particle suffers a displacement $r$ during the time interval $t$.

The lateral distinct van Hove function

$$
g_{d}(r, t)=\frac{1}{n}\left\langle\frac{1}{N} \sum_{\substack{i, j=1 \\ i \neq j}}^{N} \delta\left(\mathbf{r}-\mathbf{R}_{i}(t)+\mathbf{R}_{j}(0)\right)\right\rangle,
$$

with $g_{d}(r, 0)=g(r)$, is proportional to the conditional probability density of finding, at time $t$, a particle a distance $r$ apart from the location of another one at an earlier time $t$ $=0$. It is thus the time dependent generalization of the twodimensional radial distribution function. Note that $g_{d}(r$ $\rightarrow \infty, t)=g_{d}(r, t \rightarrow \infty)=1$ in the fluid phase. Q2D quantities like $g_{s}(r, t), g_{d}(r, t)$, and $W(t)$ can be conveniently measured for micron-sized colloidal particles using video microscopy imaging. 2,3

The space-time properties $g_{s}(r, t)$ and $g_{d}(r, t)$ are essentially the Fourier transform pairs of the self-intermediate scattering function

$$
G(q, t)=\left\langle e^{i \mathbf{q} \cdot\left[\mathbf{R}_{i}(t)-\mathbf{R}_{i}(0)\right]}\right\rangle=n \int d^{2} r e^{i \mathbf{q} \cdot \mathbf{r}_{s}} g_{s}(r, t)
$$

and of the distinct part

$$
\begin{aligned}
S(q, t)-G(q, t) & =\left\langle\frac{1}{N} \sum_{\substack{i, j=1 \\
i \neq j}}^{N} e^{i \mathbf{q} \cdot\left[\mathbf{R}_{i}(t)-\mathbf{R}_{j}(0)\right]}\right\rangle \\
& =n \int d^{2} r e^{i \mathbf{q} \cdot \mathbf{r}} g_{d}(r, t)
\end{aligned}
$$

of the dynamic structure factor $S(q, t)$, respectively. The function $S(q, t)$, with $S(q, t=0)=S(q)$, is the key property determined in dynamic light scattering experiments.

The influence of HI on the exponential short-time decay of $S(q, t)$ is contained in the so-called hydrodynamic function $H(q){ }^{31,34}$ Without $\mathrm{HI}$ acting between the particles, $H(q)=1$, whereas a nonconstant $H(q)$ is a signature of hydrodynamically interacting particles. In two dimensions, $H(q)$ is given within the leading far-field (i.e., Oseen) approximation of $\mathrm{HI}$ by ${ }^{13,21}$

$H(q)=1+3 \frac{C}{q \sigma}+3 \frac{C}{\sigma} \int_{0}^{\infty} d r[g(r)-1]\left[2 J_{0}(q r)-\frac{J_{1}(q r)}{q r}\right]$

with a first-order pole at $q=0$ which is independent of the pair potential. Here, $C=\pi n a^{2}$ is the area fraction of particles. As discussed in Refs. 21 and 13, the divergence of the 
TABLE I. Parameters of the quasi-two-dimensional superparamagnetic aqueous system I and charged systems II and III without HI, and with pair potential given in Eqs. (1) and (2), respectively. The Bjerrum length, $L_{B}$ $=7.2 \AA$ corresponds to water as solvent at room temperature. The coupling parameters are $\Gamma_{\mathrm{I}}=\beta \mu_{0} M^{2} n^{3 / 2} /(4 \pi)$ and $\Gamma_{\mathrm{II}, \mathrm{III}}=L_{B} Z^{2} n^{1 / 2}$, respectively.

\begin{tabular}{lccc}
\hline \hline & System I & System II & System III \\
\hline$C=\pi n a^{2}$ & 0.02 & 0.01 & 0.003 \\
$r_{0} / a$ & 12.5 & 17.7 & 32.4 \\
$\Gamma$ & 3.9 & 47.8 & 91.4 \\
$k=\kappa r_{0}$ & $\ldots$ & 2.5 & 3.9 \\
$r_{m} / r_{0}$ & 0.98 & 0.98 & 0.97 \\
$-\left.\beta r_{0} \frac{d u}{d r}\right|_{r=\kappa^{-1}}$ & $\ldots$ & 112 & 420 \\
\hline
\end{tabular}

short-time transport coefficient $H(q)$ at $q=0$ is due to transversal confinement and due to the absence of off-plane motion. There is consequently no divergence of $H(q)$ in the three-dimensional case. Strong experimental evidence for the divergence of $H(q \rightarrow 0)$ has been found in Refs. 35 and 36.

\section{RESULTS AND DISCUSSION}

Essentially three different types of systems, labeled as I-III, are analyzed with parameters listed in Table I. System I is an aqueous magnetic Q2D system described by the pair potential in Eq. (2), whereas systems II and III are two different examples of aqueous charge-stabilized Q2D dispersions characterized by the pair potential in Eq. (1). These systems are strongly repulsive particle dispersions with pronounced next neighbor shells and masked excluded volume interactions, characterized in their static behavior by a single length scale given by the geometric mean particle distance $r_{0}$.

The radial distribution function, $g(r)$, of these systems reveals a pronounced principal peak located at a radial dis-

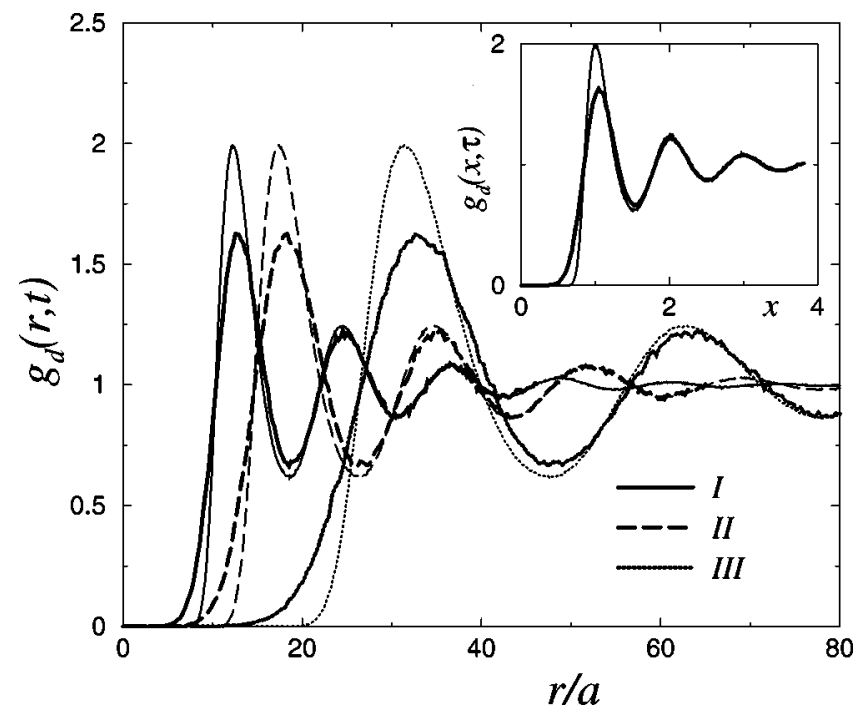

FIG. 1. BD results without HI for the distinct van Hove function $g_{d}(r, t)$ of systems I-III (cf. Table I) vs $r / a$ at $t=0$ (thin lines), where $g_{d}(r, 0)$ $=g(r)$, and at $t / \tau_{0}=0.032$ (thick lines). The characteristic times $\tau_{0}$ of I-III are different from each other. Inset: $g_{d}(x, \tau)$ vs dimensionless distance $x$ $=r / r_{0}$ and reduced time $\tau=t / \tau_{0}$, with $r_{0}=n^{-1 / 2}$ and $\tau_{0}=\left(n D_{0}\right)^{-1}$.

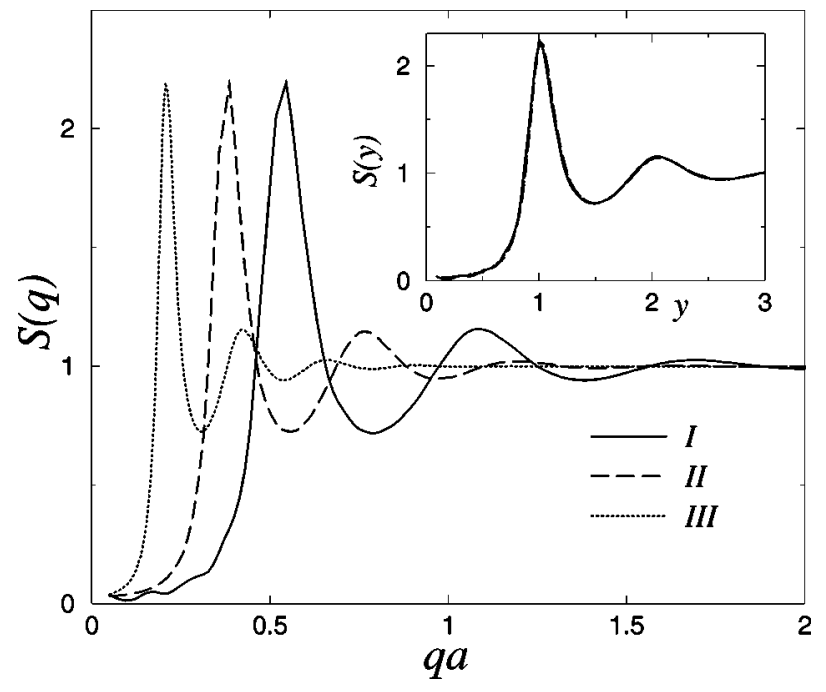

FIG. 2. Static structure factor $S(q)$ of systems I-III vs $q a$. Inset: $S(y)$ vs reduced wave number $y=q / q_{0}$.

tance $r_{m}$ which is nearly equal to $r_{0}$ (cf. Table I and Fig. 1). The system parameters have been selected such that the three $g(r)$ 's have the same principal peak height $g\left(r_{m}\right)$. When plotted versus the reduced distance $x=r / r_{0}$, the $g(r)$ 's are seen from the inset of Fig. 1 to superimpose nearly perfectly within the depicted $x$ range. This static scaling behavior of the $g(r)$ 's implies that the corresponding two-dimensional static structure factors $S(q)$, defined by Eq. (9), nearly coincide when plotted versus the reduced wave number $y$ $=q / q_{0}$ (cf. Fig. 2 and inset). Here, $q_{0}=2 \pi / r_{0} \approx q_{m}$, with the principal peak of $S(q)$ located at $q_{m}$.

Provided that $r_{m} \approx r_{0}$ is the only physically relevant length scale also for the particle dynamics, as expected without considering $\mathrm{HI}$, there is then a single characteristic time scale $\tau_{0}=r_{0}^{2} / D_{0}$ associated with $r_{0}$. Dynamic properties like the dimensionless distinct and the self van Hove functions $g_{d}(r, t)$ and $g_{s}(r, t)$ and the lateral mean squared displacement $W(t)$ depend on $r$, in case of $g_{d}$ and $g_{s}$, and on $t$ only through the reduced distance $x$ and time $\tau=t / \tau_{0}$. We note that $g_{s}(r, t), g_{d}(r, t)$, and $W(t)$ can be conveniently measured using colloidal video microscopy. ${ }^{2,3,17}$ BD results without HI for $g_{d}(r, t)$ of systems I-III at time $t=0.032 \tau_{0}$ are shown as thick lines in Fig. 1, in comparison with $g_{d}(r, 0)$ $=g(r)$ (thin lines).

Corresponding BD results without $\mathrm{HI}$ for the reduced self van Hove function $g_{s}(r, t)$ of systems I-III at $t$ $=0.032 \tau_{0}$ are displayed in Fig. 3, together with the Gaussian approximation form of $g_{s}(r, t)$ given by the right-hand side of Eq. (11). The Gaussian approximation is seen to be excellent for the shown $r$ range, illustrating that non-Gaussian corrections to $g_{s}(r, t)$ are very small at shorter times.

The BD results for the corresponding normalized selfdiffusion function $D(t)=W(t) / D_{0} t$, with $D(0)=1$, vs reduced time $\tau$ are shown with and without HI in Fig. 4. The insets in Figs. 1, 3, and 4 clearly demonstrate dynamic scaling, according to which colloidal systems with strong and long-range particle repulsion and identical peak heights $g\left(r_{m}\right)$ [likewise, identical $S\left(q_{m}\right)$ ] have nearly identical dynamical properties, e.g., nearly identical $g_{d}(r, t)$ and $W(t) / r_{m}^{2}$, as functions of reduced distance and time. More- 


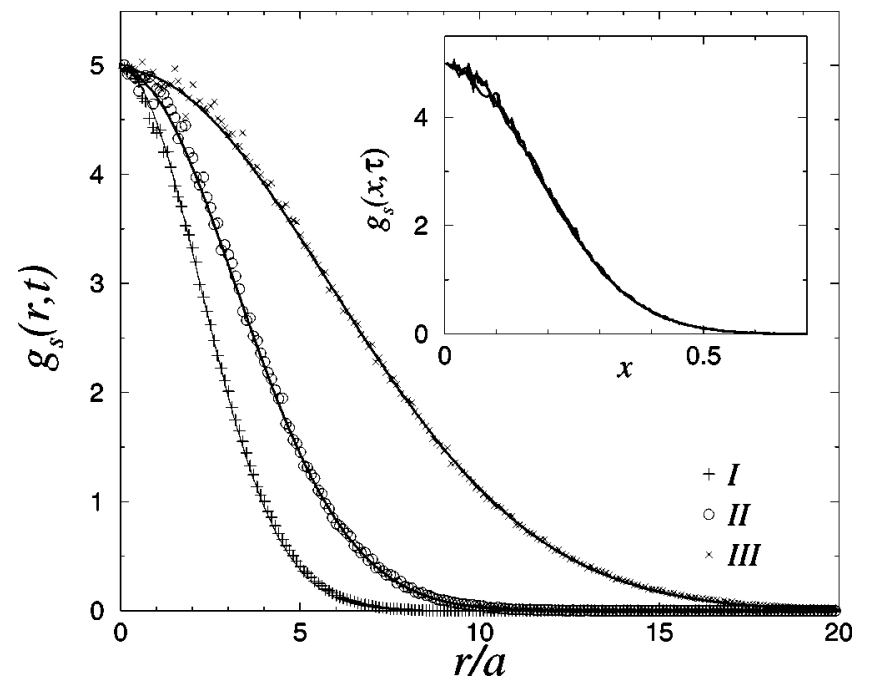

FIG. 3. BD results without $\mathrm{HI}$ for the self van Hove function $g_{s}(r, t)$ of systems I-III vs $r / a$ at $t / \tau_{0}=0.032$ (symbols). Inset: $g_{s}(x, \tau)$ vs $x=r / r_{0}$. Further shown are the Gaussian approximations for $g_{s}(r, t)$ and $g_{s}(x, \tau)$ (solid lines).

over, the dynamic structure factors $S(q, t)$ are nearly identical for these systems as a function of reduced wave number $y$ and time $\tau$.

Although dynamic scaling appears as an expected feature of systems characterized by a single relevant static length scale, it is by no means a trivial finding. Dynamic scaling of $S(q, t)$ as a function of $y$ and $\tau$ has been predicted very recently for three-dimensional de-ionized bulk dispersions of charge-stabilized particles using a mode coupling approximation (MCA) scheme with HI neglected. ${ }^{37}$ While the dynamic scaling predictions of the MCA scheme apply also to $q$-space dynamic properties in two dimensions, the present Q2D simulation study provides the first unambiguous validation of dynamic scaling, particularly regarding the involved approximations made in the MCA scheme.

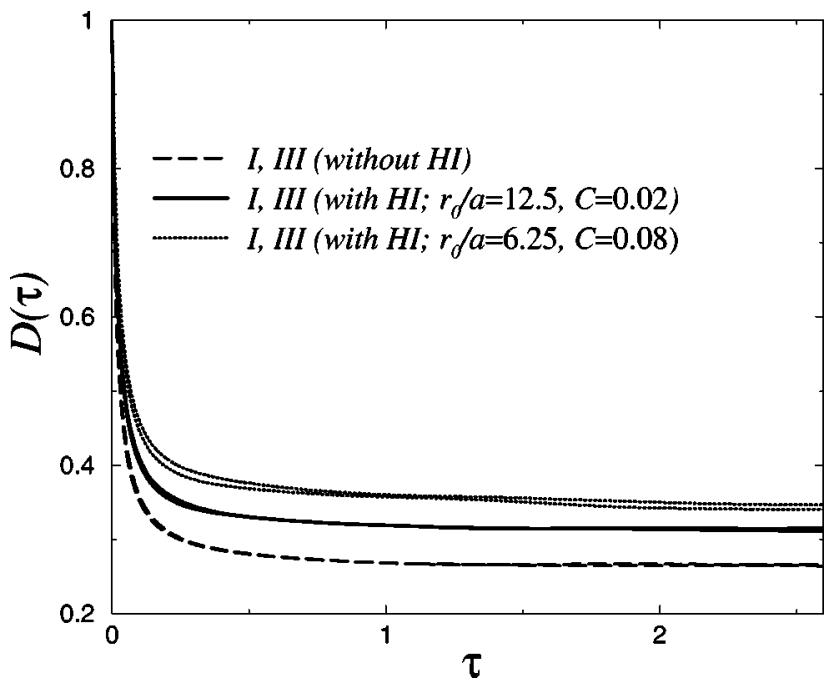

FIG. 4. Normalized self-diffusion function $D(t)=W(t) /\left(t D_{0}\right)$ of systems I and III with/without HI vs reduced time $\tau$. With HI, the system parameters are as in Table I, aside from $r_{0} / a$ and surface fraction $C$ which are given in the figure. We consider $r_{0} / a=12.5$ and 6.25 , with $S\left(q_{m}\right)=2.3$.
While the dipolar potential in Eq. (2) provides no characteristic length scale, the Yukawa-type potential in Eq. (1) includes $\kappa^{-1}$ as an intrinsic length. However, the screening lengths of the charge-stabilized systems II and III are significantly smaller than $r_{m}$ (cf. Table I) and, since $-\left.r_{0} \beta(d u / d r)\right|_{r=\kappa^{-1}} \gg 1$, with pronounced next neighbor shells occurring at distances $r_{0}$, the lengths $\kappa^{-1}$ are of no relevance regarding the static and dynamic scaling behavior (cf. Table I). The pair potentials in Eqs. (1) and (2) are special cases of the more general potential form

$$
\beta u(r)=A r^{-l} f(\kappa r)
$$

with a regular function $f$, coupling amplitude $A$, and exponent $l$. The Ornstein-Zernicke equation associated with the $g(r)$ for the potential in Eq. (16) is given in terms of reduced distance $x$ and time $\tau$ as ${ }^{38}$

$g(x)-1=c(x)+n r_{0}^{2} \int d^{2} x^{\prime} c\left(\left|\mathbf{x}-\mathbf{x}^{\prime}\right|\right)\left[g\left(x^{\prime}\right)-1\right]$,

where $c(x)$ denotes the direct static correlation function. For very large pair separations $r \gg r_{0}$,

$$
c(x) \approx-\beta u(x)=-\Gamma \frac{f(k x)}{x^{l}}
$$

with reduced dimensionless coupling parameter $\Gamma=A / r_{0}^{l}$ and reduced screening parameter $k=\kappa r_{0}$. Since $r_{0} \approx r_{m}=n^{-1 / 2}$ holds to very good accuracy for the dispersions under consideration, it follows from Eqs. (17) and (18) that systems of equal $\Gamma=A n^{l / 2}$ and $k=\kappa n^{-1 / 2}$ are statically equivalent, i.e., they have identical pair functions $c(x)$ and $g(x)$. Likewise, the generalized Smoluchowski equation for the $N$-particle probability density $P\left(\mathbf{R}^{N}, t\right)$, which is statistically equivalent to Eq. (3) without HI, is expressed in terms of reduced position vectors $\mathbf{X}_{i}=\mathbf{R}_{i} / r_{0}$ and $\tau$ as ${ }^{31}$

$\frac{\partial}{\partial \tau} P\left(\mathbf{X}^{N}, \tau\right)=\sum_{i=1}^{N} \frac{\partial}{\partial \mathbf{X}_{i}} \cdot\left[\frac{\partial}{\partial \mathbf{X}_{i}}+\beta \frac{\partial}{\partial \mathbf{X}_{i}} U\left(\mathbf{X}^{N}\right)\right] P\left(\mathbf{X}^{N}, \tau\right)$,

where $\mathbf{X}^{N}=\left\{\mathbf{X}_{1}, \ldots, \mathbf{X}_{N}\right\}$ and $U\left(\mathbf{X}^{N}\right)=\Sigma_{i<j}^{N} u\left(\left|\mathbf{X}_{i}-\mathbf{X}_{j}\right|\right)$. It follows readily from Eqs. (17) and (19) that systems with equal $\Gamma$ and $k$ are also dynamically equivalent. According to Table I, the values for $\Gamma$ and $k$ in systems I-III are all different from each other. This means that the static and dynamic scaling observed in Figs. 1-4 is not just a straightforward consequence of the special forms of the potentials in Eqs. (1) and (2). Since $c(x) \approx-\Gamma f(k x) / x^{l}$ for $x \gg 1$, there are in fact differences in the small wave number behavior of $S(y)$ for systems I-III, and hence in the thermodynamic properties. However, due to the smallness of $S\left(q \ll q_{0}\right) \ll 1$ for strongly repulsive particle dispersions, these differences are visible only when $n \hat{c}(y)=1-1 / S(y)$ is plotted vs $y$ instead of $S(y),{ }^{37}$ where $\hat{c}(y)$ denotes the Fourier transform of $c(y)$.

The dynamic scaling for quasi-two-dimensional dispersions with strong long-range particle repulsion implies, in particular, that systems of equal $g\left(r_{m}\right)$ and $S\left(q_{m}\right)$ also have the same value of the normalized long-time self-diffusion coefficient $D_{S}^{L}=\lim _{t \rightarrow \infty} W(t) / t$. The one-to-one correspondence between $S\left(q_{m}\right)$ and $D_{S}^{L} / D_{0}$ is displayed in Fig. 5, with 


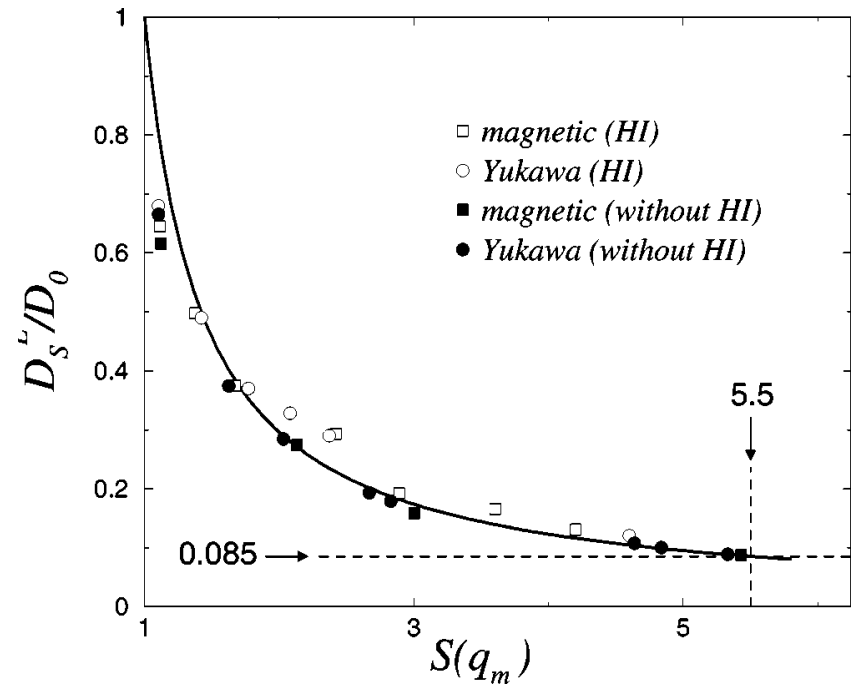

FIG. 5. Reduced long-time self-diffusion coefficient $D_{S}^{L} / D_{0}$ without HI (closed symbols) and with far-field HI (open symbols, for $r_{0} / a=6.25$ ) vs liquid static structure factor peak height $S\left(q_{m}\right)$. System parameters are as for I and III, aside from $\Gamma$ and $k$ which are varied. Solid line: parametrization of the relation $D_{S}^{L} / D_{0}=f\left(S\left(q_{m}\right)\right)$, using the form $f(z)=0.42 /(z-0.58)$ with $f(1)=1$ and $f(5.5)=0.085$, which holds approximately for $z \geqslant 1.5$.

$D_{S}^{L}$ extrapolated from large- $t$ BD calculations of $W(t)$ as described in Ref. 39. Without HI, the BD results for $D_{S}^{L} / D_{0}$ vs $S\left(q_{m}\right)$ of both the magnetic and Yukawa systems are located on a single master curve (cf. Fig. 5), in particular with a value of $D_{S}^{L} / D_{0} \approx 0.085$ for the peak height $S\left(q_{m}\right) \approx 5.5$. According to an empirical dynamical criterion for twodimensional freezing found by Löwen ${ }^{39}$ from BD simulations without $\mathrm{HI}$ for various inverse-power pair potentials, $D_{S}^{L} / D_{0} \approx 0.085$ at the freezing line, independent of $u(r)$ and the nature of the freezing process. Moreover, the peak height $S\left(q_{m}\right)$ of the liquid static structure factor at two-dimensional freezing was found to be about 5.5 at the freezing line, ${ }^{40}$ in good accord with the BD results in Fig. 5. For comparison, the three-dimensional Hansen-Verlet rule ${ }^{41}$ of freezing gives a smaller value for $S\left(q_{m}\right)$ of about 2.85. This indicates the more strongly pronounced static correlations in twodimensional fluids close to freezing. In contrast, the value of $D_{S}^{L} / D_{0} \approx 0.098$ for the onset of three-dimensional freezing ${ }^{37,39}$ is rather close to the one in two dimensions.

Summarizing, we have shown that the equivalence of two seemingly unrelated static $^{40}$ and dynamic ${ }^{39}$ criteria for two-dimensional freezing is a special consequence of dynamic scaling. Due to dynamic scaling, there is also a one-to-one correspondence, e.g., between $S\left(q_{m}\right)$ and the socalled long-time collective diffusion coefficient $D_{C}^{L}\left(q_{m}\right)$ $=-q_{m}^{-2} \lim _{t \rightarrow \infty}(d / d t) \ln \left[S\left(q_{m}, t\right)\right]$ (cf. Ref. 37). The unique value of $D_{C}^{L}\left(q_{m}\right) / D_{0}$ at the freezing point can be equally well used in place of $D_{S}^{L}\left(q_{m}\right) / D_{0}$ as an indicator for the onset of freezing.

So far we have disregarded the dynamical influence of HI, which introduces the particle radius $a$ as another relevant length scale since the spheres are in contact with the solvent. For the pairwise-additive far-field part of the hydrodynamic diffusivity tensor prevailing for the systems under consideration, this fact becomes apparent from noting that
$\mathbf{D}_{i j}\left(\mathbf{R}^{N}\right) / D_{0} \approx \mathbf{D}_{i j}^{(2)}\left(\left[\mathbf{R}_{i}-\mathbf{R}_{j}\right] / a\right) / D_{0} \cdot{ }^{42,43}$ With HI, there thus exists a more restricted form of dynamic scaling where the master curves for $g_{d}(x, \tau)$ and $D(\tau)$ depend on the ratio $a / r_{0}$. For particles diffusing in the midplane between two parallel walls, $\mathbf{D}_{i j}$ depends of course also on the ratio $h / r_{0}$. However, we consider the wall distance to be fixed here. This restricted dynamic scaling behavior can be deduced also from MCA results for Brownian systems with far-field HI included ${ }^{10}$ which relate the memory function associated with $S(q, t)$ to $S(q)$ and to the hydrodynamic function $H(q)$ defined in Eq. (15). Within the leading far-field (i.e., Oseen) approximation of HI, the two-dimensional $H(q)$ is given in reduced variables by ${ }^{21}$

$$
\begin{aligned}
H(y)= & 1+\frac{3}{4}\left(\frac{a}{r_{0}}\right) \frac{1}{y}+\frac{3 \pi}{2}\left(\frac{a}{r_{0}}\right) \int_{0}^{\infty} d x[g(x)-1] \\
& \times\left[2 J_{0}(y x)-\frac{J_{1}(y x)}{y x}\right]
\end{aligned}
$$

with a first order pole at $y=0$. Equation (20) shows explicitly the dependence of $H(y)$ on the ratio, $a / r_{0}$, of the dynamic and static characteristic lengths.

BD results for $D(\tau)$ and $D_{S}^{L} / D_{0}$ vs $S\left(q_{m}\right)$ with HI included are depicted in Figs. 4 and 5, respectively. In Fig. 4, two size ratios $r_{0} / a=6.25$ and $r_{0} / a=12.5$ are used to show the dependence of $D(\tau)$ on $r_{0} / a$. The observed modest enhancement of self-diffusion, indicative of prevailing far-field $\mathrm{HI},{ }^{10,12,13,17}$ becomes stronger with decreasing ratio $r_{0} / a$ (cf. Fig. 4). The value of $D_{S}^{L} / D_{0}$ close to freezing is modestly enlarged by $\mathrm{HI}$ as anticipated from Fig. 4 for the case $r_{0} / a$ $=6.25$. BD simulations with $\mathrm{HI}$ become exceedingly time consuming when the freezing point is approached since a large number of particles is needed to account for the strong and long-range particle correlations. Note that the so-called short-time self-diffusion coefficient $D_{S}^{S}=\lim _{t \rightarrow 0} W(t) / t$ is equal to $D_{0}$ for systems with prevailing far-field HI. In contrast, for systems with dominating near-field $\mathrm{HI}$ and strong lubrication forces like dispersions of colloidal hard spheres, $D_{S}^{S}<D_{0}$, the dynamic freezing criterion must then be stated in terms of $D_{S}^{L} / D_{S}^{S}$ instead of $D_{S}^{L} / D_{0}$, as has been shown for three-dimensional bulk systems by forced Rayleigh scattering experiments ${ }^{44}$ and by rescaled MCA calculations. ${ }^{37} \mathrm{BD}$ results for $H(q)$ of systems I and II with HI treated in the point-force (Oseen) and Rotne-Prager approximation, respectively, are included in Fig. 6. The restricted dynamic scaling behavior of the short-time property $H(y)$ (cf. the inset of Fig. 6) and its pole at $y=0$ can be clearly observed. The BD results for $H(y)$ in the point-force approximation are indistinguishable from the Rotne-Prager results, indicating that it is sufficient to account for the leading order term of the far-field HI only.

To conclude, we have analyzed the static and dynamic scaling behavior of strongly repulsive Q2D dispersions with and without HI. Dynamic scaling was shown for these systems to be at the origin of the equivalence of two freezing criteria for the onset of two-dimensional freezing. Further extensions of this work will address possible consequences of dynamic scaling on higher order static and dynamic correlation functions with more than two particle coordinates 


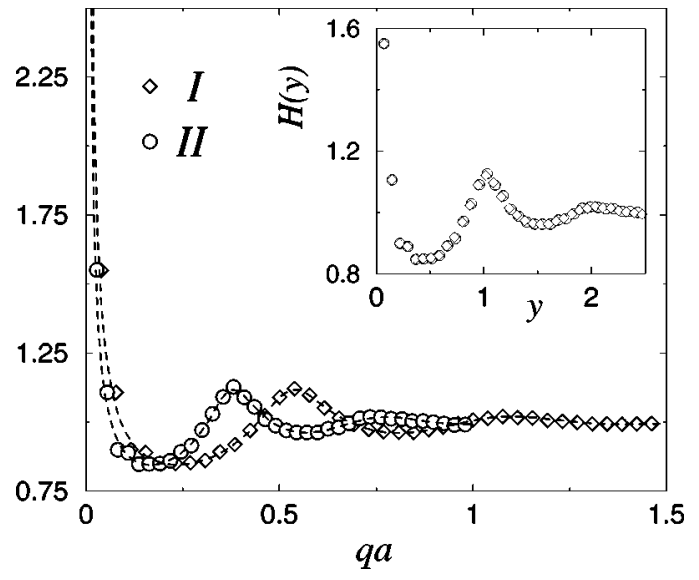

FIG. 6. Two-dimensional hydrodynamic function $H(q)$ of systems I and II in point-force (symbols) and Rotne-Prager (dashed lines) approximations. Inset: $H(y)$ of systems I and II vs reduced dimensionless wave number $y$ $=q r_{0}$. System parameters are as in Table I for I and II, aside from $r_{0} / a$ $=6.25$ and $C=0.08$ used here.

involved. Moreover, we plan to investigate possible connections with a corresponding states relationship for transport coefficients introduced by Rosenfeld. ${ }^{45}$

\section{ACKNOWLEDGMENTS}

We gratefully acknowledge very helpful support in part of the BD simulation coding by B. Rinn and P. Maass, and we thank J. K. G. Dhont and R. Klein for their interest in this work. This research work was funded by the Deutsche Forschungsgemeinschaft (SFB 513).

${ }^{1}$ L. Lobry and N. Ostrowsky, Phys. Rev. B 53, 12050 (1996).

${ }^{2}$ H. Acuña-Campa, M. D. Carbajal-Tinoco, J. L. Arauz-Lara, and M. Medina-Noyola, Phys. Rev. Lett. 80, 5802 (1998).

${ }^{3}$ A. E. Larsen and D. G. Grier, Nature (London) 385, 230 (1997).

${ }^{4}$ K. Zahn and G. Maret, Phys. Rev. Lett. 85, 3656 (2000).

${ }^{5}$ K. J. Strandburg, Rev. Mod. Phys. 60, 161 (1988).

${ }^{6}$ D. Goulding and J.-P. Hansen, Europhys. Lett. 46, 407 (1999); Mol. Phys. 95, 649 (1998).

${ }^{7}$ E. Allahyrov, I. D'Amico, and H. Löwen, Phys. Rev. E 60, 3199 (1999).

${ }^{8}$ T. M. Squires and M. P. Brenner, Phys. Rev. Lett. 85, 4976 (2000).

${ }^{9}$ R. Pesché and G. Nägele, Europhys. Lett. 51, 584 (2000).

${ }^{10}$ G. Nägele and P. Baur, Physica A 245, 297 (1997); Europhys. Lett. 38, 557 (1997).
${ }^{11}$ W. Härtl, J. Wagner, Ch. Beck, F. Gierschner, and R. Hempelmann, J. Phys.: Condens. Matter 12, A287 (2000).

${ }^{12}$ B. Rinn, K. Zahn, P. Maass, and G. Maret, Europhys. Lett. 46, 537 (1999).

${ }^{13}$ R. Pesché and G. Nägele, Phys. Rev. E 62, 5432 (2000).

${ }^{14}$ E. Chang and D. W. Hone, Europhys. Lett. 5, 635 (1988).

${ }^{15}$ B. Löhle and R. Klein, Physica A 235, 224 (1997).

${ }^{16}$ H. Löwen, J. Phys.: Condens. Matter 4, 10105 (1992).

${ }^{17}$ K. Zahn, J. M. Méndez-Alcaraz, and G. Maret, Phys. Rev. Lett. 79, 175 (1997).

${ }^{18}$ D. L. Ermak and J. A. McCammon, J. Chem. Phys. 69, 1352 (1978).

${ }^{19}$ J. Rotne and S. Prager, J. Chem. Phys. 50, 4831 (1969).

${ }^{20}$ J. Happel and H. Brenner, Low Reynolds Number Hydrodynamics (Noordhoff International, Leyden, 1973).

${ }^{21}$ A. J. Banchio, Ph.D. thesis, University of Konstanz, Germany, 1999.

${ }^{22}$ S. H. Lee and L. G. Leal, J. Fluid Mech. 98, 193 (1980).

${ }^{23}$ E. R. Dufresne, D. Altman, and D. G. Grier, Europhys. Lett. 53, 264 (2001).

${ }^{24}$ R. B. Jones, B. U. Felderhof, and J. M. Deutch, Macromolecules 8, 680 (1975).

${ }^{25}$ K. Aderogba and J. R. Blake, Bull. Aust. Math. Soc. 18, 345 (1978).

${ }^{26}$ B. Cichocki, R. B. Jones, R. Kutteh, and E. Wajnryb, J. Chem. Phys. 112, 2548 (2000).

${ }^{27}$ G. Bossis, A. Meunier, and J. D. Sherwood, Phys. Fluids A 3, 1853 (1991).

${ }^{28}$ A. Meunier, Ph.D. thesis, University of Nice, France, 1990.

${ }^{29}$ E. R. Dufresne, T. M. Squires, M. P. Brenner, and D. G. Grier, Phys. Rev. Lett. 85, 3317 (2000).

${ }^{30}$ C. W. J. Beenakker, J. Chem. Phys. 85, 1581 (1986).

${ }^{31}$ G. Nägele, Phys. Rep. 272, 215 (1996).

${ }^{32}$ B. R. A. Nijbor and A. Rahman, Physica (Amsterdam) 32, 415 (1966).

${ }^{33}$ J. Schofield, A. H. Marcus, and S. A. Rice, J. Phys. Chem. 100, 18950 (1996).

${ }^{34}$ W. Härtl, Ch. Beck, and R. Hempelmann, J. Chem. Phys. 110, 7070 (1999).

${ }^{35}$ B. Lin, S. A. Rice, and D. A. Weitz, J. Chem. Phys. 99, 8308 (1993).

${ }^{36}$ B. Lin, S. A. Rice, and D. A. Weitz, Phys. Rev. E 51, 423 (1995).

${ }^{37}$ A. J. Banchio, G. Nägele, and J. Bergenholtz, J. Chem. Phys. 113, 3381 (2000).

${ }^{38}$ J. P. Hansen and I. R. McDonald, Theory of Simple Liquids (Academic, New York, 1986).

${ }^{39}$ H. Löwen, Phys. Rev. E 53, R29 (1996).

${ }^{40}$ J. Q. Broughton, G. H. Gilmer, and Y. D. Weeks, Phys. Rev. B 25, 4651 (1982).

${ }^{41}$ J. P. Hansen and L. Verlet, Phys. Rev. 184, 151 (1969).

${ }^{42}$ S. Kim and S. J. Karrila, Microhydrodynamics: Principles and Selected Applications (Butterworth-Heinemann, London, 1991).

${ }^{43}$ J. K. G. Dhont, An Introduction to Dynamics of Colloids (Elsevier, Amsterdam, 1996).

${ }^{44}$ H. Löwen, T. Palberg, and R. G. Simon, Phys. Rev. Lett. 70, 1557 (1993).

${ }^{45}$ Y. Rosenfeld, Phys. Rev. E 62, 7524 (2000). 